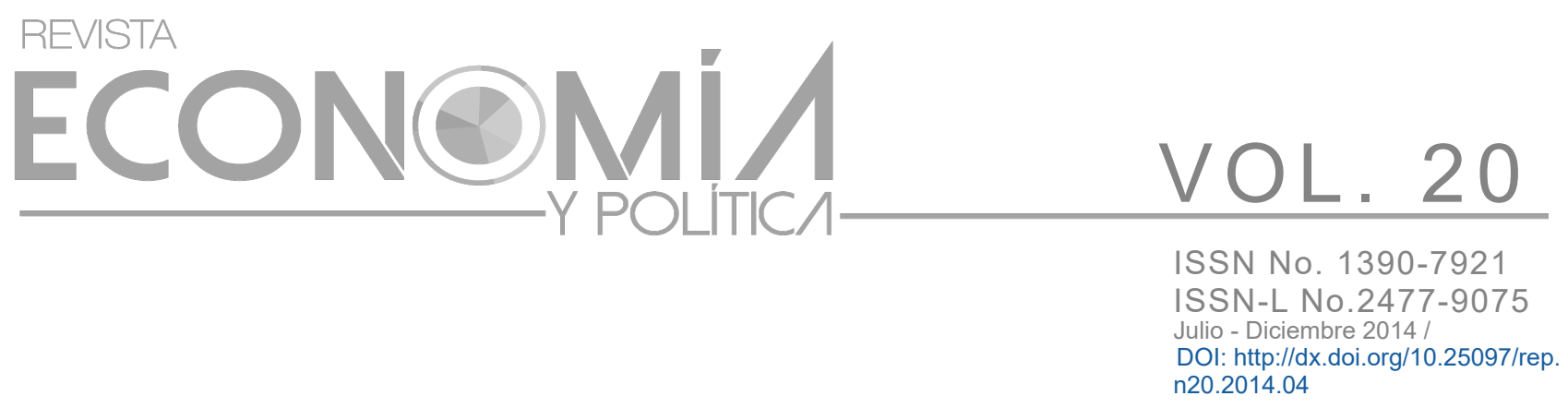

\title{
REDES DESARTICULADAS Y CAMBIO TECNOLÓGICO. ASENSO Y OCASO DE NOKIA COMO INNOVADOR EN EL MERCADO DE TELÉFONOS MÓVILES.
}

\section{DISJOINTED NETWORKS AND TECHNOLOGICAL CHANGE. CONSENT AND DECLINE OF NOKIA AS AN INNOVATOR IN THE MOBILE PHONES MARKET.}

\section{Matías Milia}

Universidad de Cuenca

matias.milia@ucuenca.edu.ec
Juan Ignacio Serenellini

Buenos Aires , Argentina.

\section{Resumen:}

Este artículo plantea un análisis posible del objeto "Sistema Operativo Móvil para teléfonos inteligentes" y su influencia en el desempeño de Nokia como empresa fabricante de teléfonos móviles. A través del análisis de caso se busca dar herramientas para pensar el fenómeno de la innovación proponiendo un acercamiento complejo a los fenómenos tecnológicos y su dimensión económica y social. Para ello recurrimos a la Teoría del Actor Red (TAR), analizando las redes de actores humanos-no humanos, los programas declarados por Nokia y los anti- programas sobre los cuales buscaba operar la empresa. Estudiamos la gestión de la innovación dentro de Nokia a través de un abordaje a la micropolítica de la innovación. Tras introducir características de la industria y su evolución tecnológica, analizamos el sistema operativo (OS) Symbian y el rol del mismo en el nuevo paradigma. Luego de avanzar sobre la influencia de Symbian Ltd en Nokia, se analiza su influencia en la postergación de Nokia en las innovaciones de telefonía celular. Para ello, se analizan las propiedades configurativas del objeto Sistema Operativo Móvil, y su impacto sobre Nokia, como habilitadoras y desarrolladoras de redes de actantes humanos y no humanos. Se discuten las relaciones entre hardware y software, como así también las uniones 'no fortuitas' de innovaciones que esto implica. Se concluye y elabora sobre la centralidad del objeto 'Sistema Operativo Móvil' en la configuración de las nuevas redes entre actores híbridos en la industria de la telefonía móvil.

Palabras clave: Innovación, Teoría del Actor Red, Micropolítica de la Innovación, Nokia, Teléfono Móvil.

\begin{abstract}
:
This article proposes a possible analysis of the object 'Mobile Operating System for smartphones' and its influence on the performance of Nokia as a mobile phone manufacturer. The analysis of this case of study intends to provide tools to think of the phenomenon of innovation, proposing a complex approach towards technology as an economic and social phenomenon. From the perspective of the Actor- Network Theory (ANT), the human-non human networks of actors are analyzed, as well as the programs declared by Nokia and the anti-programs over which the company tried to oparate. The management of innovation is studied within Nokia through an approach towards micropolitics of innovation. After introducing industry characteristics and its technological developments, the Operating System (OS) Symbian and its role in the new paradigm are analyzed. The influence of Symbian Ltd. in Nokia and its setback in mobile phone innovation is discussed. The configurative properties of the object 'Mobile Operating System', and its impact on Nokia, are addressed as facilitating and developing tools to expand and reform human-non human actant networks. The relationships between hardware and software are studied, as well as the 'not random' links whitin different implied innovations. At last, it is concluded and elaborated over the importance of the object 'Mobile Operating System' in the configuration of the new networks between hybrid actors in the mobile phone industry.
\end{abstract}

Key Words: Innovation, Actor-Network Theory, Innovation Micropolitics, Nokia, Cell Phone. 


\section{Introducción}

Desde concluída la Segunda Guerra Mundial, con la adaptación de tecnologías militares a las necesidades civiles se colocan a la ciencia y la tecnología como bases para una innovación que, validada en mercados y sociedades, ha logrado construir riqueza y sustentar la promesa de un mayor y mejor bienestar para los seres humanos. Parte de estas mejoras a la vida en el planeta han estado relacionadas, en los últimos 30 años, a la industria de las telecomunicaciones.

Sin embargo, la historia de la tecnología está repleta de paradojas, fracasos estrepitosos y notorias disrupciones. Nuevas concepciones de las ciencias sociales eliminan la distinción entre actores humanos y no humanos (Latour B. , 2008[2005]; 1983; 1998) para el análisis de la tecnología, su rol social y económico. Estructurado en torno a la comprensión del ascenso y ocaso de una de las principales y más simbólicas- compañías de teléfonos móviles, Nokia, nos proponemos brindar herramientas conceptuales para comprender los procesos de nacimiento y consolidación de tecnologías innovadoras. Trabajaremos articulando nuestra exposición sobre el objeto 'teléfono móvil' y las variaciones de las relaciones construidas en torno al mismo entre actores híbridos (Latour B. , 2008[2005]).

Estas distintas fases estables darán lugar a tres distintos 'momentos' que hemos logrado identificar para el desarrollo, posicionamiento y percepción del objeto 'teléfono móvil'. Se describirá cómo Noka participó de las relaciones y propuso 'programas' (Latour B. , 1998) para la configuración de una red que, en cada fase, consiguiera la estabilidad y 'repetición' de conductas y elecciones por sobre otras posibles alternativas existentes (Latour \& Lépinay, 2009[2008], pp. 53-57). Mediante una descripción de la empresa, su entorno, se introducirán dos actores híbridos de singular importancia Symbian Ltd.y el sistema operativo homónimo. Sobre el final, se conceptualizará el ocaso de Nokia dentro de la industria, a partir de los cambios en el objeto 'teléfono movil', la red de actores y la micropolítica de la innovación (Jones \& Stevens, 1999) en el eje Nokia-Symbian.

El período de estudio va del año 1960 hasta 2010 concentrándose entre los años 1998 y 2007, años de ascenso y ocaso de la red que contaba con Nokia y Symbian Ltd. como actantes privilegiados. Se han establecido hitos entre el período 1960-2010 que, si bien responden a momentos importantes en el desarrollo del teléfono móvil, fueron elegidos para favorecer la historización del proceso y la construcción del relato.

La historia del teléfono móvil puede dividirse en tres momentos: una fase inicial "mecánica", caracterizada por la búsqueda de estabilización del objeto a partir de las características tecnológicas, una segunda fase, la fase de "popularización" donde la inestabilidad tecnológica ya fue resuelta y la tecnología y la reinterpretación social del objeto 'teléfono móvil' dan lugar a una rápida adaptación y masificación del uso público de la tecnología (Agar, 2003; Farley, 2005; 2013; Lindholm, 2003) y finalmente, la fase más cercana, la fase de "fusión" donde se sintetizan en el teléfono móvil distintos dispositivos electrónicos a través de aplicaciones y este se vuelve una plataforma para el consumo y producción de información multimedia (Reed, 2010).

\section{Fase Electromecánica}

La fase electromecánica sobrevuela la década del sesenta hasta finales de la década del ochenta del Siglo XX. Las primeras redes de telefonía celular comerciales y experimentales a escala media fueron construidas en Escandinavia a principios de la década del sesenta. La configuración primigenia de las relaciones entre actores humanos y no humanos $(\mathrm{H}-\mathrm{NH})$ construida en torno al objeto 'teléfono móvil' tuvo durante esta fase un 
bajo grado de estabilización tecnológica o 'robustes' (Woolgar, 1998). El teléfono móvil estuvo signado durante el período por un contexto de búsqueda de soluciones sociotécnicas principalmente desde el punto de vista mecánico-electrónico en lo técnico y regulatorio-comercial desde lo económico-social.

Mientras que en EE.UU. las diferencias regulatorias entre estados y los intereses de las empresas existente en otras areas del mercado desalentaron el desarrollo y homologación de esta tecnología, en Europa la mayor preocupación se centró en resolver el problema técnico que significaban la portabilidad del teléfono móvil y la adaptación del teléfono móvil a la multiplicidad de redes y protocolos (Farley 2005; 2011). Las capacidades adaptativas tanto de protocolos, como de soluciones técnicas y de producción de las firmas escandinavas adquiridas en otros campos de las telecomunicaciones, como la digitalización de líneas telefónicas por parte de Nokia en los setentas, sería central para configurar eficientemente las redes $\mathrm{H}$ - NH y la correcta traducción de la tecnología de telefonía móvil (Agar, 2003).

La complejidad geopolítica en la que este primer estado de la red vio la luz en el norte de Europa preparó a los fabricantes para la competencia en el resto del mundo. Los primeros avances en tecnologías de roaming y homologación de normas fueron los fundamentales. Luego, un consorcio entre fabricantes y telefónicas europeas promulgaría la norma GSM ${ }^{1}$, el primer paso hacia la consolidación de una 'caja negra' tecnológica (Lehne, 2010).

La seguridad y estabilidad que brindaron las normas desarrolladas permitirían una aplicación en otros territorios, ampliando la aceptación de esta tecnología digital. La cadena de actores que se constituiría en torno a esta elección técnica sería lo suficientemente larga y amplia para ganar cada vez mayores 'estados de realidad' (Latour 1998). Este sería el primer aporte para consolidar el nuevo objeto, el teléfono celular. El segundo sería dado por Nokia con la fabricación en serie de los primeros teléfonos bajo estándares digitales ${ }^{2}$. La paulatina consolidación de percepciones compartidas de atributos técnicos y protocolos ayudó a configurar los distintos usuarios (Woolgar et al. 1998) y sus expectativas para con la tecnología móvil.

\section{Fase de Popularización}

En la fase de popularización se llegará por primera vez a un alto grado de estabilidad en el sintagma de actores humanos y no humanos en torno al teléfono móvil. Con la "caja negra" del teléfono móvil cerrada en torno la tecnología electrónica y los distintos estándares de transmisión digital se logró una nueva configuración avanzando hacia la masificación del uso de los teléfonos celulares. La estabilización de la tecnología potenció la adopción de los teléfonos móviles a gran escala.

El dominio de la tecnología digital con que contaba Nokia, sumado a la amplia base de sustento a los nuevos estándares, le dio una posición de privilegio en la segunda fase. La empresa se constituiría como un privilegiado 'enunciador del programa' o actante (Latour 1998:131) de esta red de H-NH. Su modelo Cityman, primero, y el primer modelo GSM el Nokia 1011, luego, configurarían los usuarios de teléfonos móviles que comenzarían a aumentar en número en todo el mundo.

Como se observa en el Gráfico 1 (GapMinder, 2013) la ampliación de esos 'grados de realidad' es evidente en el aumento considerable de usuarios dentro de los países

\footnotetext{
${ }^{1}$ Global System for Mobile Communications u, originalmente Groupe Spécial Mobile.

${ }^{2}$ Vale destacar que como plantea (Agar, 2013) Nokia se insertaba en el contexto finés de fines de la guerra fría lo que alentaba la toma de decisiones más drástricas ante el fin del equilibrio comunismo-capitalismo del que el país se había beneficiado tanto.
} 
industrializados que se sucede a partir de 1992. La idea planteada por Latour (1998:125) de que los actores involucrados en una innovación co- evolucionan nos ayudará a entender las implicancias de estos momentos en tanto que los usuarios, las empresas telefónicas, los fabricantes se irán co-produciendo uno a otro. En tanto se logra construir esta 'caja negra' y reducir los niveles de incertidumbre es que podemos hablar que en este período los usuarios logran ser 'usuarios configurados' (Woolgar 1998). Lo significativo es aquí el gran aumento en la llamada 'distancia social' entre los productores de esta tecnología y los usuarios, reduciendo los niveles incertidumbre de los últimos.

Efectivamente al aumentar el tamaño del mercado el crecimiento de los intermediarios ayuda a construir la traducción necesaria (Latour 1998) para consolidar esta tecnología. Como explica Woolgar (1998:580) la tarea de los intermediarios será central en la construcción de la llamada 'brecha de certeza', más profunda la brecha más sólidas son las innovaciones, algo clave para construir la percepción de los atributos técnicos del objeto.

La red tendida en relación al teléfono móvil consta de cuatro grandes ramas agrupando a los fabricantes, usuarios, intermediarios y el tándem entre estándares-empresas telefónica-infraestructura-regulaciones. Los fabricantes tienen en la red una posición como actantes privilegiados desarrollan nuevas soluciones en sus laboratorios de I+D basándose en mejoras electrónicas que permiten la miniaturización, mejora de recepción y mayor eficiencia de uso de batería a la vez que 'interpretan' las necesidades de los usuarios. La empresas fabricantes diseñan sus propios equipos, desarrollando proveedores en microchips y baterías. Estas relaciones se configuran bajo las directivas de los fabricantes, que se vuelven el nodo central de la red.

Los usuarios se caracterizan por portar la lógica de los early adopters, descritos como miembros de la sociedad con mayores ingresos, de mediana edad y con experiencia con dispositivos tecnológicos similares (Dickerson \& Gentry, 1983; Frank, 2001), y comienza a manifestarse la tendencia a la incorporación sostenida de nuevos usuarios no vinculados directamente al teléfono celular como herramienta de trabajo. Intermediarios como los medios de comunicación, la cultura popular y la publicidad van construyendo un entramado simbólico que comienza a articular la elaboración del 'sentido' de este dispositivo y su contexto (Woolgar, 1998, p. 72).

Por su parte, la estrecha relación construida entre las empresas telefónicas, los estándares de trasmisión, las regulaciones gubernamentales y la infraestructura tecnológica, obraron difundiendo el programa de los fabricantes de equipos. Las empresas que diseñaban y construían los teléfonos móviles eran quienes dominaban la tecnología necesaria también para permitir a estos otros actores satisfacer sus intereses. Esta configuración de la red de actores $\mathrm{H}-\mathrm{NH}$ sería la base de la explotación del negocio de la telefonía celular por algo más de diez años y permitiría la consolidación económica de los principales fabricantes de terminales que tendieron a desarrollar estos vínculos para enriquecer sus programas de acción y mejorar su rentabilidad y ganancias.

Un rasgo central de esta fase es la aparición de los sistemas operativos en los teléfonos (Agar, 2003). Los sistemas operativos se concentraban fundamentalmente en aspectos referidos al hardware como por ejemplo la optimización del uso de la batería y la interconexión entre kernel y las diferentes partes del teléfono. Por otro lado, el sistema operativo era la base para el diálogo correcto entre el teléfono móvil y la red operadora del servicio.

Los OS incorporaron durante este período crecientes funcionalidades. Los productores de terminales, como Nokia o Motorola, exigirán más allá de los requerimientos básicos a sus 
sistemas operativos para incorporar al usuario masivo cada vez más demandante a medida la brecha con la tecnología móvil disminuía.

A mediados de la década de los noventa comienzan a hacer su aparición los dispositivos PDA (Personal Digital Assistant) siendo de alguna forma la contracara de los teléfonos celulares: son el primer punto de contacto entre el mundo de las computadoras y el mundo de móvil y de portabilidad. Los PDA, sin la limitación obligada de la comunicación telefónica, se concentraron en ampliar los 'features' y las funcionalidades del sistema operativo en un dispositivo móvil. Es por ello que el nombre que recibe la terminal en este período es "teléfono utilitario" porque permitía incorporar herramientas y 'utilidades' aunque no lograba mayores avances en el procesamiento, generación ni trasmisión de información.

Es en este período que hace su aparición Symbian OS en 1998, el primer sistema operativo móvil (OS) para terminales en el marco de una unión de Ericsson, Nokia, Motorola y Psion para explotar la convergencia entre PDA (más conocidos como PALMs) y los teléfonos móviles. Ese mismo año el hito de la estabilidad de esta etapa salió al mercado, el Nokia 1100 , la terminal y el dispositivo electrónico más vendido de la historia.

\section{Fase de Fusión}

El crecimiento de la demanda de terminales volvió a dibujar una exponencial a partir de finales de la década del noventa con la expansión física de la tecnología y redes GSM.

En la fase de fusión, entre los años 2003 a 2008 como se comienza a superar la barrera de un teléfono por habitante, como vemos en el Gráfico 2 (GapMinder, 2013). Asimismo, publicaciones de la época (Van Thanh, Jørstad, \& Jonvik, 2005) discutían sobre el éxito de las dos primeras grandes aplicaciones: la navegación por internet y el e-mail.

En la fase de fusión confluyen varios procesos y aquí nuevamente la caja negra se abre y el futuro del teléfono móvil comienza a ser impreciso. Se puede hablar de la computadorización de los teléfonos o la telefonización de las computadoras. Paralelamente, los dispositivos tendieron a concentrar cada vez más funciones hasta entonces tradicionalmente dispersas en otros aparatos. Este fenómeno estuvo sobre todo orientado a los dispositivos de captura y reproducción de sonido, video e imágenes. También se iría sumando, paulatinamente, la función del entretenimiento con juegos electrónicos y otros pasatiempos. De esta forma se comenzaron a tender las relaciones que facilitarían la unificación de estas redes e irían configurando al objeto teléfono móvil.

Lo central es que en la fase de fusión converge el mundo de los dispositivos multimedia, las computadoras, el mundo de la Internet y el mundo de la telefonía móvil. Esta fase que había comenzado a gestarse con los PDA toma forma con la incorporación de la internet móvil a los teléfonos celulares a través de la tecnología WAP para transferencia de datos y luego la tecnología $2 \mathrm{G}$ y $3 \mathrm{G}$.

Este hecho no constituye más que el resultado de otras variaciones en los humanos y no humanos que se suceden simultáneamente. La nueva relación de los grupos de actores de geometría variable se relacionarían con el objeto 'teléfono celular' de geometría variable también (Latour 1998:124-125).

Esta nueva configuración del teléfono celular tendría implicancias técnicas, comerciales y económicas para los distintos actores involucrados. En lo técnico, el eje se centraría ya no en soluciones a problemas electrónicos específicos de la telefonía o en miniaturización de componentes sino bajo una creciente estandarización de partes entre los dispositivos electrónicos bajo el paraguas de la portabilidad informática permitió la incorporación de nuevos jugadores al mercado y nuevos actores a la red. A la red se sumarían de manera 
paulatina nuevos actores con experiencia y capacidades heredadas del mundo de internet, el software y las computadoras como Apple, Microsoft o Google.

Así se configurarían nuevas relaciones con la inclusión los servicios basados en internet como el correo electrónico, la transferencia de datos y los distintos browsers. El rol del sistema operativo en este período es fundamental y con el sistema operativo surgen tres actores no humanos de vital importancia para las sucesivas interpretaciones que se hagan del teléfono móvil: las Uls -user interfaces- o interfaces gráficas y, por otro lado, la experiencia de usuario y finalmente las API (aplicaciones).

Por el lado de los actores humanos se incorporaron a la red todos los actores que pertenecían a la red de la Internet: distintos generadores de contenido como diarios, revistas, radios y canales de TV. La nueva forma de articulación de estas relaciones ya no sería centralmente sobre el objeto 'teléfono celular' si no sobre la plataforma de los 'sistemas operativos' debido a que los sistemas operativos y la forma en que las compañías que los desarrollan operan sobre ellos determinará la creación de ecosistemas propios del sistema operativo que incorporarán a todos los nuevos actores relacionados con el desarrollo de contenido.

Los sistemas operativos son a la fase de fusión lo que el hardware era a la fase de popularización: un punto de pasaje obligatorio. El OS determinará el grado de extensión de la red y la complejidad de las interacciones entre los actores. Como nuevos actantes de la red que habilitaron algunos OS se incorporan son los developers (desarrolladores). Gracias a las particularidades de los sistemas operativos como el Apple OS que dejan una libertad de actuación grande, los developers fueron liberados del control de las compañías y fue durante este período que desarrollaron aplicaciones para las terminales móviles.

El nombre que recibe el objeto de estudio 'teléfono móvil' en este período es "smartphone" o "teléfono inteligente". La co-evolución de los usuarios y las tecnologías para prestar los servicios de telefonía fueron dándose correlativamente. De igual forma, durante este tercer momento es cuando se produce una nueva estandarización tecnológica en la que la eficiencia y la rentabilidad estarían basadas en otras prestaciones de los equipos.

En este momento de la historia del objeto teléfono móvil vemos como se incorporan cada vez más elementos de otras redes. Las innovaciones acumuladas por años en campos como la fotografía digital, la reproducción y almacenamiento de archivos multimedia, el desarrollo de video juegos, comenzarían a confluir en torno a este objeto. Como explica Latour (1998:126) 'La dominación no es nunca un capital que pueda ser almacenado en un banco. Debe ser desplegado, cajanegrizado, reparado, mantenido.'. La posición dominante de los fabricantes de teléfonos se vió alterada ante la necesidad de construir nuevas lealtades y traducirlas en una nueva 'caja negra'.

La inclusión del sistema operativo trajo aparejada la necesidad de fijar plataformas para el desarrollo de las aplicaciones que fueran dinámicas, interesantes y atrayentes para los desarrolladores más talentosos. La incorporación de nuevas funcionalidades como audio, video o juegos obligó a las empresas a abrir su 'caja negra' a nuevas empresas especializadas en esas áreas. Las corporaciones telefónicas, por su parte, ganaron peso y poder de negociación al transformarse en uno de los canales privilegiados para la venta de equipos (Reed, 2010). También las telefónicas pudieron echar mano de sus capacidades técnicas y comerciales adquiridas en la venta de servicios de conexión a internet. Esto obligó a las empresas fabricantes a construir alianzas que permitieran dar 'mantenimiento' a estas relaciones. Ejemplo resonante fue la alianza entre la japonesa Sony y la sueca Ericsson. Otras empresas, como Samsung, lograrían 
aprovechar las oportunidades de colaboración con fabricantes consolidados para construir equipos que irían ganando sostenidamente espacio en el mercado.

La puesta en juego de ciertas 'capacidades dinámicas' (Eisenhardt y Martin 2000) por parte de las empresas como Apple o Google dio por resultado un cambio en la administración, división y apropiación de los distintos recursos incorporados en la red. Esta correcta lectura de las nuevas transformaciones potenció el rol de algunos actantes.

Más adelante exploraremos cómo la incorrecta lectura de la fase de "fusión" llevó a Nokia a dilatar su propia flexibilidad interpretativa (Pinch, 2008) del objeto de estudio. Además, se intentará explicar a través de la micropolíticade la organización Nokia y Symbian Ltd (Jones \& Stevens, 1999) cómo el proceso de desarrollo de producto se vio desviado hacia interpretaciones erróneas de la tendencia que realizaban ya el mercado, los competidores y los consumidores a través de sus demandas.

\section{Nokia, Symbian y la Fase de Fusión}

\section{a. Un perfil de Nokia Corporation}

Nokia Corporation es una corporación de origen finlandés de telecomunicaciones que pertenece a Nokia Corporation. Nokia está involucrada en la manufactura de dispositivos móviles y en la convergencia de Internet y las industrias de la comunicación. Con más 132.000 en 120 países y ventas en más de 150 países, Nokia tiene un revenue global anual por encima de los 42 billiones de euros. Es el manufacturador más grande de teléfonos móviles con una participación de 23\% de Mercado en el Segundo cuarto de 2011 (Nokia Corporation, 2011; Gartner, 2011).

Nokia supo comenzar la primera década del Siglo XXI con más del $43 \%$ de participación como manufacturador de teléfonos móviles. Nokia es una compañía que, desde su fundación en 1865, realizó diversas adaptaciones de su negocio. Sus productos móviles se caracterizaron por la calidad del hardware por sobre otras particularidades. Más allá de avanzar en la miniaturización de sus componentes para favorecer la portabilidad, Nokia pudo dar mejor autonomía de batería y mejor calidad de recepción en sus primeras terminales que sus rivales.

Podemos decir que Nokia supo traducir, reinterpretar y producir una terminal sólida, estabilizando la tecnología, conquistando la confianza de los usuarios,

'disminuyendo el margen de negociación' y logrando efectivamente cerrar la caja sobre los teléfonos móviles (Latour 1998:126). Esta estabilización permitió la rápida masificación del producto. Esta configuración de los usuarios al 'modo Nokia' le permitió establecerse como benchmark de otras compañías a la hora de producir y diseñar dispositivos móviles.

En lo corporativo fue un actor central para alinear a la red al momento de establecer protocolos, tal como lo fue en el caso del establecimiento de la red de GSM (AMPLIADO) en el que cumplió un papel protagónico dentro del escenario europeo. Su manejo dominante de las tecnologías digitales volcadas a las telecomunicaciones le otorgó la ventaja necesaria para influir en la norma estandarizaciones, ampliando su rol preponderante como eje central de la red de innovadores.

Podemos decir que, según las fases delimitadas en este trabajo, el éxito de Nokia fue en la fase de popularización. La concentración de Nokia en el hardware relegó el desarrollo de su software, siendo este actor no humano central en el estado actual de las redes tendidas en torno al teléfono móvil.

La forma de desarrollo de terminales móviles y la interacción del software y hardware según Nokia, estaban dominadas por los requerimientos del hardware. Los ajustes y 
programaciones del software se ajustaban a los pedidos de los ingenieros del hardware. Las adaptaciones de las interfaces gráficas, que luego devendrían en Uls estaban dominadas por las limitaciones del hardware e incluso los features del teléfono se ceñían a no entorpecer las funciones centrales del teléfono: la duración de la batería y la calidad de la comunicación.

Esta acción y decisión a la hora de diseñar las terminales deja entrever la traducción (Latour B. , 1983) que hacía Nokia del objeto y nos permite ver, a la luz de la historia actual, que Nokia había traducido al teléfono móvil justamente como su nombre lo indica como un teléfono por sobre todas las cosas y móvil porque podía ser desplazado. Estos dos aspectos serían las fronteras del objeto.

Esta definición del objeto fue muy exitosa pero sin embargo cerró la puerta a nuevas traducciones que la siguieron, hechas fundamentalmente por los usuarios más configurados y que vendrían de la mano de la 'computadorización' del teléfono móvil: el teléfono móvil para muchos usuarios no era un teléfono exclusivamente, era un 'dispositivo móvil' y, por lo tanto tenía abierta la puerta a nuevos espacios de traducción y reproducción.

Volviendo a la fase de popularización, Nokia supo leer claramente las necesidades de los usuarios (Lindholm y Keinonen 2003) con las nuevas demandas de los consumidores, agregando a tiempo features a sus terminales tal como lo hacían las compañías japonesas, chinas y norteamericanas pero manteniendo sus dos bastiones: uso de batería y conectividad.

La cristalización de este período fue el Nokia 1011, primer teléfono GSM producido, lanzado al mercado en 1992 que permitía la trasferencia de datos a través de mensajes SMS. Pero estos nuevos teléfonos móviles requerían nuevas características de software más avanzadas que las que permitía el sistema operativo disponible y, por ello, fue necesario desarrollar Symbian OS.

\section{b. Symbian OS}

A medida que avanzaba la última década del Siglo $X X$, con los desafíos de protocolos y hardware ya estabilizados, las terminales móviles continuaron desarrollando sus interfaces gráficas y operativas para sus usuarios. Era este el momento en que las sucesivas traducciones (Latour B. , 1983) realizadas por los consumidores de los teléfonos móviles comenzaron a exigir al teléfono móvil más prestaciones. La lógica era bastante sencilla para las empresas y para los consumidores: la dependencia de la portabilidad sumado a las capacidades técnicas del hardware dotaban al teléfono móvil de una flexibilidad interpretativa amplia (Pinch, 2008, p. 84); era un teléfono móvil, pero también un reloj, un cronómetro, un calendario portable, una alarma. El teléfono móvil adquirió, como diría Pinch "significados en el mundo social y estos significados dan forma y constriñen el desarrollo de una tecnología". Continua Pinch: "A menudo, en las etapas iniciales del desarrollo de una tecnología se desarrollan diferentes significados de una tecnología, algunos enfrentados entre sí.”. En línea con Pinch, se dio cierta oposición entre los teléfonos móviles y los PDA.

Los PDAs contaban con todos estos features que se les empezaba a exigir a las terminales móviles. La fusión entre estos dispositivos -primer intento de fusión entre el mundo de las computadoras y el mundo de las terminales móviles- era inevitable ${ }^{3}$. En este contexto

\footnotetext{
${ }^{3}$ Para los teléfonos móviles era imperioso, dentro de los requerimientos de esta fusión, contar con un sistema operativo que ya no solo se encargara del hardware sino que hiciera a las veces de soporte de features más
} 
comienzan las negociaciones entre los principales productores de teléfonos móviles, liderados por Nokia, para formar la compañía de software que respondiera a las necesidades de todos los productores de teléfonos móviles.

En junio de 1998 se crea Symbian Ltd, una empresa de desarrollo y emisora de licencias de software como un partnership entre Ericsson, Nokia, Motorola, and Psion para explotar la convergencia de los PDA y los teléfonos móviles. Es interesante remarcar la dispar participación accionaria de los shareholders en Symbian Ltd: Nokia poseía el $56.3 \%$ de las acciones, Ericsson el $15.6 \%$, Sony Ericsson $13.1 \%$ y otros productores se repartían el restante $16 \%$.

Cada una de las compañías pertenecientes a Symbian Ltd eran licenciatarios del software, es decir, debían pagar a Symbian Ltd por el uso del software y en contraposición Symbian Ltd se comprometía a desarrollar y adaptar el software para distintas necesidades del productor o del carrier de telefonía de cada país. Visto de este modo, Symbian era una enorme avance porque evitaba a los licenciatarios tener que ajustar su software particular a cada mercado y cada carrier y podían aprovechar la escala del desarrollo que había Symbian Ltd para todos. Desde una óptica estratégica Symbian Ltd era una maniobra y apuesta óptima para garantizar a los licenciatarios ser first movers en los mercados donde los licenciatarios quisieran participar y tomar la ventaja por sobre otros productores de terminales. Symbian era una forma "cerrar" mercados a este pseudo cartel de licenciatarios a través de una estandarización tecnológica.

\section{c. Nokia, Symbian Ltd, Symbian OS y la micropolítica de la innovación}

Frente a un contexto tan ambicioso de expansión, e inminente reformulación de los teléfonos móviles, ¿por qué una empresa como Nokia, experimentada y con muchos años en la industria de las telecomunicaciones, no pudo llevar a su sistema operativo Symbian OS a ser, como lo habían sido sus teléfonos móviles, benchmark y referente del mercado?

¿Por qué Nokia hoy, diez años después de la fundación de Symbian Ltd decidió abandonar Symbian OS y entregar su sistema operativo a Microsoft Corp en 2011? ¿Qué sucedió en el medio? ¿Cuál fue la dinámica de la convergencia entre los PDA y las terminales móviles que dejó afuera del concierto de interpretaciones vencedoras a Nokia? ¿Cómo se amplió la red luego de la fusión entre el mundo de las computadoras y el mundo de los teléfonos móviles inteligentes? Las respuestas pueden encontrarse mucho más cerca de la micropolítica de desarrollo de Nokia que sobre las condiciones del "mercado".

Como hemos señalado anteriormente en el trabajo, los sistemas operativos -y no el hardware- ganaron protagonismo luego que se abriera la caja negra de esta tecnología a los teléfonos móviles inteligentes. El paso de 'teléfonos móviles funcionales' a 'teléfonos móviles inteligentes' estableció al software como punto de pasaje obligatorio y a la vez fue un habilitador de un nuevo ecosistema de redes donde ganaron protagonismo los desarrolladores de software. La dinámica de incorporación de los actores nuevos al ecosistema -y los programas que opusieron cada una de las compañías participantesfueron determinantes a la hora de definir y cerrar hasta el día de hoy los teléfonos móviles inteligentes.

El desarrollo de Symbian Ltd y la influencia de la micropolítica de desarrollo de este software nos sirven para comprender en profundidad como el prometedor avance resultó en

complejos, interfaces gráficas más sofisticadas (UI), que incorporara la visión del usuario (usabilidad) y el uso de teclados como el Nokia 9210 Communicator. 
un fracaso. El intento de Nokia por protocolizar el OS mediante la articulación centralizada de los actores implicados en la producción, tal como lo había hecho con el protocolo GSM años, se transformó en uno de los más exitosos fracasos de la historia tecnológica (Ockock, 2010). Tim Ocock, ingeniero, trabajó en Symbian Ltd entre 1998 y 2004 en roles técnicos y comerciales. Symbian era a comienzos de la primera década del Siglo XXI “...el sistema operativo más grande por participación de mercado, el más avanzado en términos de economía de batería y el de menor requerimientos de recursos del software." (Ockock, 2010).

En la red de Symbian Ltd se encontraban actores heredados de la configuración de teléfono inteligente ${ }^{4}$ y también actores de Internet ${ }^{5}$.

El principal problema que encuentra Ocock (2010) sobre Symbian era que no era un sistema operativo para Internet, que debe tener un buen browser y "...el browser siempre fue un ciudadano de segunda clase, un componente tercerizado.". Lo que importaba eran las necesidades del hardware o de los ingenieros de hardware.

El segundo problema consistía en la dificultad para programar sobre Symbian. Era esta dificultad el primer programa de Nokia para mantener el control absoluto sobre el contenido. Symbian tenía una reputación de ser una plataforma sobre la cual era difícil programar dados sus orígenes como adaptación de EPOC ${ }^{6}$ para PDAs y su particular forma de programación en $\mathrm{C}++$, junto a las adaptaciones y cambios a los standards ANSI. Esto no ayudaba a construir programas base para la programación. La situación descripta "causaba mucha frustración a los (programadores) recién llegados a la plataforma por la "verbosidad" de los códigos de Symbian" (Ockock, 2010).

La segunda carga en esta línea era la Accredited Symbian Developer (ASD, Desarrollador de Symbian Acreditado), una acreditación de renovación anual para el desarrollo de software para desarrollos de Symbian OS que determinaba la relación entre la empresa y los desarrolladores, haciendo las veces de llavero pesado y grande unido a una llave de hotel, para no olvidar quién es el dueño de ella y recordar dejarla en recepción al salir (Latour B. , 1998, p. 132).

Sin embargo, la escritura de buen código Symbian era beneficiosa para Symbian Ltd. y hacía que quisiera seguir manteniendo las cosas tal como estaban: el $80 \%$ de sus ingresos provenían de licenciatarios en forma de consultoría y desarrollo. Con esta estructura "...no había incentivos a producir [desde Symbian Ltd] un sistema operativo un sistema operativo eficiente." según Ocock (Ocock, 2010). Así, la empresa se garantizaba, a pesar de depender de sus licenciatarios, ser un punto de paso obligatorio para todas las compañías productoras de terminales. Pasar por Symbian significaba que los tiempos de producción de cada nueva terminal se midieran en años y no meses como hoy en día con Android.

En resumen, Symbian Ltd y Nokia impusieron una idiosincrasia a la red de actores alineándola detrás de las necesidades de Symbian Ltd como organización Nokiadependiente y como una organización concentrada en intereses más financieros que de calidad. Este intento de protocolización y aprovechamiento de las economías de escala a través de un único OS para todas las compañías resultó, tal como estaba diseñado, en un sistema de lock-in que retrasó a todas las compañías y además, dados los programas

\footnotetext{
${ }^{4}$ Estos son: productores de terminales, carriers telefónicos, ingenieros de hardware, ingenieros de software, usuarios, dentro de Nokia particularmente líderes de producto, dentro de Symbian Ltd su CEO y CFO y los licenciatarios.

${ }^{5}$ Aquí se pueden ver a desarrolladores de aplicaciones, nuevos desarrolladores de software, compañías de Internet, fabricantes de computadoras, entre otros.

${ }^{6}$ Sistema Operativo antecesor de Symbian y base del mismo.
} 
en el sentido latouriano del término- y distintas cargas de Symbian en su OS impidieron incorporar una red de actores nueva que surgió de la fusión entre la telefonía móvil inteligente, los PDA e Internet.

Como resultado de este retraso tecnológico que representó Symbian OS existió un enorme espacio, de mercado y temporal, para el desarrollo de empresas como Apple o Google con capacidades distintas al resto de los productores de terminales. Estas capacidades eran, fundamentalmente, estar habituados a desarrollar software a través de terceros e incluirlos en sus desarrollos, unificándolos al software.

Se puede argumentar decir entonces, a modo de conclusión, que Nokia y Symbian Ltd no contaban con las capacidades dinámicas (Eisenhardt, 2000) que sí contaron 6 años después sus competidores Apple y Google con Apple OS y Android respectivamente, para coexistir con otros actores que participaran de la fase popularización, para coexistir con actores que ellos no pueden controlar sino apenas dirigir e incentivar.

Los teléfonos inteligentes de hoy en día, a través de actores no humanos como Google Play o Appstore permiten alinear y articular los intereses de desarrolladores y productores de Uls y aplicaciones en línea con los objetivos de Apple o Google. Estas aplicaciones, a diferencia de las de Symbian, se cuentan en millares y a Apple o Google no le insumen ningún costo y reportan ganancias. La sucesión de programas de Nokia versus los antiprogramas de programadores impusieron dentro de Symbian OS diferentes candados para que se haga lo que Nokia quería y, como comenta Andrew Orlowski (2011) 'se desperdiciaran 2.000 años hombre en Uls y aplicaciones que no funcionaron'. En cotraste, hoy en la telefonía móvil, la red no tiene actores humanos (o corporativos) si no híbridos como punto de pasaje obligatorio: está articulada en torno a los sistemas operativos, el software y las subredes que estos actores habilitan.

\section{Conclusiones}

No es una tarea sencilla predecir el desarrollo tecnológico dentro de los ambientes corporativos en industrias de tecnología, no es una tarea fácilmente historizable y muy difícilmente pueda establecerse un sendero de evolución predecible no solo para la tecnología en sí sino también para el negocio/corporación como un todo.

Con el caso Nokia expuesto hasta aquí, queda claro que no existen fórmulas para ideales para el liderazgo, que no existen métodos mejores que otros. Ni la cultura, ni "the Nokia's way" ("a la forma de Nokia") son soluciones eficientes frente a la falta de certeza.

Libros y artículos publicados como "Winning Across Global Markets. How Nokia creates strategic advantage in a fast changing world" de Dan Steinbock (2010), no hacen más que resaltar la que incertezas existentes sobre el progreso del desarrollo de corporaciones tecnológicas dependientes de sus inversiones en I+D.

En el caso de Nokia, también es muy difícil decir, a la luz de 1998, que la convergencia entre teléfonos móviles y PDAs iba a terminar anulando a ambos y como resultado de la convergencia se impondría cierto tipo de dispositivo móvil que combinaba características de ambos y además incorporaría a Internet y a las computadoras personales.

Desde la teoría popular de management es también muy difícil de desaconsejar las apuestas que hizo Nokia: intentar protocolizar el software a través de Symbian y hacer converger a todos los productores de terminales bajo un sistema que Nokia controlaría eventualmente por participación política mayoritaria. Una maniobra de convergencia y protocolización era muy similar a la que había realizado años antes frente a la convergencia de redes GSM y que le había garantizado el éxito comercial por una década. 
Contratar al mejor equipo en Symbian Ltd en 2001 para que desarrollaran tecnologías que verían la luz diez años después está en línea teorías de "masa crítica" (Rogers, 1971) para generar innovaciones superadoras.

Incluso una aproximación desde la óptica de Porter (1985) de 'ventajas competitivas' como la que realiza Steinbock (2010) nos señala que Nokia -aun en esa fecha- tiene una posición superior para competir en un mercado global de rápido cambio por su trabajo en equipo, su capacidad de adaptación a nuevos mercados, por el conocimiento de los clientes y por sobre todas las cosas, su cultura; ventajas únicas y propias de Nokia, superiores a la competencia, sostenibles en el largo plazo y adaptables a diferentes situaciones de mercado.

Aproximaciones del estilo popular del management frente al caso Nokia desconocerían la trayectoria tecnológica (Latour B. , 1998) de la innovación y se concentrarían en aspectos estéticos de la organización. Citando a D. Shivakumar, miembro del top management de Nokia, a través de Steinbock (2010, p. 47): "el fuerte trabajo en equipo reduce las tensiones internas y políticas. (...)Los 'nokians' decimos que tenemos menos intrigas políticas o fricción interdepartamental que caracteriza a muchas otras compañías. La compañía está concentrada en lograr objetivos (achievement) y ganar, algo que es buscado a través de una forma comprometida y con integridad."

Desde una óptica menos tradicional del management podemos decir que Nokia fue muy eficiente y abierta a incluir a los usuarios, a los lead users (Von Hippel, 1976) dentro del proceso de desarrollo, adaptándose a sus exigencias insatisfechas e incorporando features (alarmas telefónicas por ejemplo) y, a la vez, reinterpretando lo que los consumidores líderes hacían (free revealing) con sus terminales co-construyendo la tecnología. También Nokia fue muy eficiente en configurar a los usuarios y reducir la distancia e incertidumbre entre ellos y la tecnología. Sin embargo, no terminan de dar una respuesta acabada a la pregunta "¿Qué hizo que Nokia descendiera de la palestra de las compañías de terminales y la industria de las telecomunicaciones?"

Algún esbozo de respuesta sobre la pregunta inicial puede encontrarse si se analizan elementos relacionados por un lado con la micropolítica de la organización (Jones \& Stevens, 1999) y con elementos de la dinámica de las redes tecno-científicas (Latour B. , 1998).

Como desarrollan Jones y Stevens (1999), muchos textos de management 'eligen ignorar' el rol de la política en el management. Las pujas políticas son muy intensas, como lo demuestra el caso de Symbian OS, en gran parte porque en gran parte las empresas tecnológicas dada su dinámica particular, el diálogo con la tecnología futura es el diálogo con los cuadros de mando futuros de la organización. Parafraseando a Foucault, quien detente "saber" detentará "verdad" y eventualmente se posicionará de frente al dominio del "poder" dentro de la organización.

El modelo lineal de desarrollo de producto de Kotler (1997 - de Jones \& Stevens 1999) sostiene que "se debe encontrar primero el problema a solucionar", sin embargo, la definición del problema esconde tras de sí una orientación estratégica con lo cual, aun tomando el proceso de New Product Developement de Kotler como cierto, sería imposible negar la existencia de la política en el proceso.

En el caso de Symbian Ltd la dinámica micropolítica de mantener el statu quo por parte de los ingenieros de hardware e ingenieros compiladores tenían claro que orientaciones hacia el software implicarían una pérdida de poder.

Como señala Frost y Egri (1990, p. 6): 
"En el caso de innovaciones en el producto, el sello de la falla está en la intensa actividad política tanto en la capa superficial como en lo profundo de la estructura jerárquica. Intereses opuestos frente a una innovación movilizan una amplia gama de tácticas para preservar el status quo."

Resultaba muy dificultoso para Nokia, siendo una organización concentrada en el hardware desde la fase mecánica y la fase de popularización, impulsar una lógica de innovación contraria a esta vía. Se explican así paradojas como el tener en 2001 en sus laboratorios de I+D avances en hardware superiores a su tiempo (touch screens, por ejemplo) para ser usadas en un software con infinitas limitaciones.

Referido a las redes tecnológicas, es interesante destacar cómo la indiferenciación entre los distintos niveles en su relación al centro. Un elemento periférico como es el software, de escasa relevancia en la fase mecánica, salta a primera fila en la fase de fusión y, en su curva ascendente, desarticula las redes del hardware. La sucesión de programas (Latour B. , 1998) incorporados dentro del sistema operativo -frente a los antiprogramas del resto de los actores- dotaron de carga a la voluntad de Nokia de mantener su sistema cerrado, precipitando como organización innovadora en términos comerciales en pocos años.

Podemos concluir a modo de cierre que en el caso del teléfono móvil, las redes tecnológicas y los actores dentro de las redes co-evolucionaron en la medida que el objeto "teléfono móvil" se ampliaba, se popularizaba, se fusionaba con otros objetos y se co-construía junto con los usuarios hacia los "teléfonos móviles funcionales" y luego hacia los "teléfonos móviles inteligentes". En el caso de Nokia y Symbian, la incapacidad de interpretar la trayectoria del objeto y las cargas que portaba, ni las redes que habilitaba y cancelaba en las distintas dimenciones fueron determinantes y suficientes como para ser dejada a un costado de las nuevas redes construidas en torno al "teléfono móvil inteligente", hoy plataforma central en el consumo y producción de información multimedia (Reed, 2010). No es sorpresivo entonces que hoy en día, las principales compañías y puntos de pasaje obligatorios sean nada menos que Apple y Google, desarrolladores de computadoras el primero y de software e Internet el segundo. 


\section{Bibliografía:}

- Agar, J. (2013). Constant touch: A global history of the mobile phone. Icon Books.

- Eisenhardt, K. M. Dynamic capabilities: What are they? Strategic Management

- Journal. Special Issue: The Evolution of Firm Capabilities. , 21 (10/11), 1105-1121.

Farley, T. (2005). Mobile telephone history. Telektronikk (3/4), 22-34.

- Farley, T. (2013, 10 10). Privateline.com: Mobile Telephone History. Retrieved from http://www.privateline.com/PCS/history9.htm Frost, P. y. (1990). Influence of political action on innovation: Part I. Leadership \& Organization Development Journal , 11 (1), 17-25.

- GapMinder. (2013, 12 20). Mobile cellular subscriptions (per 100 people). En base a datos del Banco Mundial. Retrieved from GapMinder: http://www.gapminder.org/data/

- GapMinder. (2013, 12 20). Internet users (per 100 people) En base a datos del Banco Mundial. Retrieved from GapMinder: http://www.gapminder.org/data/

- Gartner. (2011, Agosto 11). [Press Release] Gartner Says Sales of Mobile Devices in Second Quarter of 2011 Grew 16.5 Percent Year-on-Year; Smartphone Sales Grew 74 Percent. Retrieved Septiembre 16, 2013, from Gartner: http://www.gartner.com/newsroom/id/1764714

- Grint, K. y. The machine at work. Chapter 3: Configuring the User: Inventing New Technologies. Cambridge, England: Cambridge Polity Press.

- Jones, O. \&. (1999). Evaluating failure in the innovation process: the micropolitics of new product development. R\&D Management , 29 (2), 167-178.

- Kotler, P. (1997). Marketing Management Analysis: Planning, Implementation and Control. Englewood Cliffs, New Jersey: Prentice Hall.

- Latour, B. (2008[2005]). Reensamblar lo social. Una introducción a la teoría del actor-red. Buenos Aires, Argentina: Manantial.

- Latour, B. y. (2009[2008]). La economía, la ciencia de los intereses apasionados. Buenos Aires, Argentina: Manantial.

- Latour, B. (1983). Give Me a Laboratory and I will Raise the World. Science Observed: Perspectives on the Social Study of Science , 141-170.

- Latour, B. La tecnología es la sociedad hecha para que dure. In M. y. Domenech, Sociología simétrica. Ensayos sobre ciencia, tecnología y sociedad. Barcelona: Gedisa. 
- Lehne, P. H. Mobile Broadband Evolution - Past, Present and Future. Telektronikk (1), 5-22.

- Lindholm, C. y. (2003). Mobile Usability: How Nokia Changed the Face of the Mobile Phone. McGraw-Hill

- Nokia Corporation. (2008). Nokia in brief-2007. Retrieved Octubre 20, 2011, from http://www.nokia.com/NOKIA_COM_1/About_Nokia/Sidebars_new_concept/Nokia _in_brief/InBriefJuly08.pdf

- Nokia Corporation. (2011). Annual Results 2010. Espoo: Nokia.

- Pinch, T. (2008). La tecnología como institución: ¿Qué nos pueden enseñar los estudios sociales de la tecnología? Revista REDES (27), 77-96.

- Porter, M. (1985). Competitive Advantage. New York: Free Press.

- Steinbock, D. (2010). Winning Across Global Markets: How Nokia Creates Strategic Advantage in a Fast-Changing World. Jossey-Bass.

- Ockock, T. (2010, November 8). Symbian OS - one of the most successful failures in tech history. Retrieved October 18, 2013, from Tech Crunch: http://techcrunch.com/2010/11/08/guest-post-symbian-os-one-of-the-mostsuccessful-failures-in-tech-history-2/

- Coldewey, D. (2011, March 10). Behind The Scenes In Symbian's Development Hell. Retrieved October 23, 2013, from Tech Crunch: http://techcrunch.com/2011/03/10/behind-the-scenes-in-symbians-developmenthell/

- Orlowski, A. (2010, November 23). Symbian, The Secret History: Dark Star. Retrieved September 30, 2013, from The Register: http://www.theregister.co.uk/2010/11/23/symbian_history_part_one_dark_star/

- Orlowski, A. (2011, March 10). Why Nokia failed: 'Wasted 2,000 man years' on Uls that didn't work. Retrieved from TheRegister: www.theregister.co.uk/2011/03/10/nokia_ui_saga/page2.html

- Van Thanh, D. y. (2005). The mobile phone: Its evolution from a communication device to a universal companion. Telektronikk (3/4), 3-21.

- Van Thanh, D., Jørstad, I., \& Jonvik, T. (2005). Reading emails on mobile phones. Telektronikk (3/4), 100-122.

- Von Hippel, E. (1976). The dominant role of users in the scientific instrument innovation process. . Research policy, 5 (3). 
- Woolgar, S. V. (1998). Abilities and competencies required, particularly by small firms, to identify and acquire new technology. . Technovation , 18 (8), 575- 
